\title{
SWIFT Trust and Distrust in STRATEgIC PARTNERING RELATIONSHIPS: KEY CONSIDERATIONS OF TEAM-BASED DESIGNS
}

\author{
Terry R. Adler \\ New Mexico State University \\ Las Cruces, New Mexico
}

\begin{abstract}
We explore the integration of trust and distrust as defined by Lewicki, McAllister, and Bies (1998) with its effects on team-based designs. A hypothetical long-term contract simulation was used to study trust and distrust through a medium-term, qualitative analysis. Results indicate that teams respond differently to trust and distrust perceptions in the development of contracts. Discussion of the Lewicki et al. (1998) model demonstrates that while irust served to loosen formalities between teams and led to higher perceived quality, the presence of distrust led to the development of competition between teams, higher inter-team accountability and lesser perceived quality.
\end{abstract}

\section{Introduction}

Organizations have not only been witness to but active participants in a major phenomena over the past twenty years: the increasing use of teams to develop and implement long-term contracts used in outsourcing organizational work (Adler, 2004; Barthelemy \& Adsit, 2003; Benfratello \& Sembenelli, 2002; Boswell, 2000; Cohen \& Bailey, 1997; Paladino, Bates \& da Silveira, 2002; Schilling \& Steensma, 2001). An assumption behind outsourcing is that trust improves communication and cooperation between teams in the accomplishment of organizational work (Boon \& Holmes, 1991; Dasgupta, 1988; Jeffries \& Reed, 2000; Mayer, Davis \& Schoorman, 1995).

While it is clear that research has indicated a need for trust in partnering relationships, the study of how trust and distrust affect inter-team based designs is clearly understudied and, thus, underdeveloped. The purpose of this paper is to contribute to our understanding of the effects of trust and distrust perceptions on inter-team processes and outcomes. Using Lewicki, McAllister, and Bies's (1998) model of trust and distrust, we studied groups in a capstone business course to more fully understand how inter-team interactions are affected by the perception of simultaneous trust and distrust.

This paper investigates two fundamental assumptions with regard to trust and distrust. First, we explore the basis for understanding how inter-team relationships are affected by simultaneous trust and distrust. Given the fast-paced nature of today's work environment, the formation of 'swift' trust and distrust implies that 
teams have to make quick decisions based on interdependence of work between teams. This study also provides a fundamental assessment of how simultaneous trust and distrust perceptions affect the formality and boundaries of inter-team interactions. Since teams are being used more frequently and for more varied types of work, an investigation of Lewicki et al.'s (1998) model is long overdue. Do trust and distrust work together as hypothesized by Lewicki et al. (1998)? This study provides insight into how teams parlay perceptions of trust and distrust into a future partner relationship.

\section{The Integration of Trust and Distrust in Long-Term Contracting}

Outsourcing typically involves some degree of negotiation between teams and research in the outsourcing of organizational work providing an intricate blend of information regarding team adaptation and communication (Adler, 2004; Bell, 1990; Helewitz, 2003; Robinson \& Volkov, 1998). Outsource teams integrate individual, team, functional and organizational norms in negotiating long-term contracts (Bazerman, 1983; Knorr \& Knorr, 1978; Lacity \& Willcocks, 1998; Niederman, Brangeay, \& Wetherbe, 1991), yet these teams are constrained by contextual factors like trust and distrust.

Teams develop long-term contracts based on perceptions of trust and distrust. The concepts of trust and distrust have been researched for over 40 years with an emphasis on the intentions and behaviors of those in which a trading relationship exists (Deutsch, 1960; Hosmer, 1995). Key to understanding trust and distrust is the fact that these concepts are two unique conditions, not opposite ends of the same continuum (Luhmann, 1979). Consequently, there are unique elements of teams that contribute specifically to both the growth and decline of trust and distrust.

Partners may collaborate, but they may also compete and this leads to simultaneous trust and distrust (Rousseau, 1995). Trust and distrust are thus defined as positive and negative expectations, respectively, of another partner's future conduct while one's organization is vulnerable in the partnership (Boon \& Holmes, 1991). These expectations are firmly established in how team members perceive a partnership with regard to the extent their own livelihood can benefit or be harmed. While team dynamics are worthy factors that affect simultaneous development of trust and distrust perceptions, most organizational contracts depend on inter-team interactions to design and fulfill organizational work (Lewicki et al., 1998). Examples of simultaneous trust and distrust perceptions are provided in Table 1 as adapted from the Lewicki et al. (1998) model.

\section{Levels of Trust and Distrust in Relational Contracting}

Early definitions of individual levels of trust are explained as a "belief in the goodness of others" (Erickson, 1950), or as "an expectancy that is held by an individual or group that the word... of another individual or group can be relied upon" (Rotter, 1980). Follow-on development of the trust concept included the 
confident expectation of the trustor that the trustee will help the trustor reach a goal in an environment of risk and uncertainty (Mayer, Davis, \& Schoorman, 1995). This environment of risk and uncertainty is also what provides the basis for trust and distrust in teams.

Zand (1972) refers to trust at the team level as generalized expectations for all team members. A key task of team members is to integrate varying individual trust and distrust expectations into a team-level perception of trust and distrust. Therefore, we define trust as one group's positive perceptions of another group's conduct while in a working relationship. Similarly, distrust is one group's negative perceptions of another team's conduct while in a working relationship (adapted from Boon \& Holmes, 1991).

The perceptions of trust and distrust form the basis for hard and soft contracting in relational contracting as described by Williamson and Ouchi (1981). The authors suggest that hard contracting is required when contract requirements are explicit. Soft contracting is used when contract terms are less specific, measurable, and clearly stated, and usually in the form of tacit agreements. The underlying premise in relational contracting is that teams choose between hard and soft contracting as a framework to do work based on their perceptions of trust and distrust. This may not be a valid assumption without careful thought on how team processes affect the development of contract requirements. Hard contracting is more precise and definitional in the terms that bound a business partnership. Soft contracting tends to be less stringent and more informal, many times succumbing to the social dynamics of the business relationship. Thus, soft contracting may have many more ambiguities in contractual terms but also more opportunities for off-contract partnering and conflict resolution. Hard and soft contracting compose two competing views on how a partnership, as a relationship, should be arranged or governed (Schilling \& Steensma, 2001; Zuckerman \& Higgins, 2002).

Consideration of trust and distrust in relational contracting is required because these conditions affect how requirements are written in the contract (Jeffries \& Reed, 2000). A long-term contract is nothing more than a medium by which the partnership is framed and subsequently governed. Teams performing the task of developing a contract based on perceptions of trust and distrust greatly influence how teams interact and whether contracts end up 'hard' or 'soft' (Adler, 2005). Since teams form and adjourn quickly and regularly, Meyerson, Weick, and Kramer (1996) suggest that organizational members develop 'swift trust' perceptions based on little time to acquire information about an individual, team or organization.

\section{Swift Trust and Distrust in Team-Based Designs}

Swift trust, a term coined by Meyerson et al. (1996), can be the result of temporary teams who are thrown together to work on important, complex tasks. Teams are typically dependent on other teams but there is little time to determine if another team has a poor past performance (Ancona \& Caldwell, 1992). As Meyerson et al. (1996) stated, in order to trust a temporary group, the members must "wade in" as opposed to waiting until experience shows if a team is trustworthy. 
Swift trust and distrust are, of necessity, formed quickly to manage issues of uncertainty, risk, and perceptions between teams. Based on the concept of temporary team-based designs, swift trust and distrust develops because of the diversity of team membership, limited history working together and task non-routineness, complexity and interdependence (Meyerson et al., 1996).

Larson and LeFasto (1989) and Mishra (1996) explain that in team-based designs, trust fosters inter-teamwork by allowing individuals to stay problemfocused, promoting more efficient communication and coordination, improving the quality of collaborative outcomes, and compensating other team members who are willing to pick up the slack if one team member fails. Within a team, members are mutually accountable for the outcomes they construct (Cohen \& Bailey, 1997) and trust would seem to be a factor that maximizes inter-team accountability. Since teams are viewed as intact social entries embedded in one or more larger social systems, inter-team based efforts typically result in performance that is greater than the sum of those team parts. Thus, the existence of trust leads to the following proposition:

Proposition 1: Inter-team relationships should be perceived as of higher quality in high trust situations.

Although trust is obviously an important consideration, there is also a place for distrust perceptions in inter-team based designs. While distrust may lead to good risk mitigation a priori, distrust perceptions may also limit communication and create a priori paranoia between teams (Adler, 2005; Lewicki et al., 1998). Based on the potential for negative aspects of distrust between teams, the following proposition is offered:

Proposition 2: Inter-team relationships should be perceived as of lower quality in high distrust situations.

Simultaneous trust and distrust perceptions create unique conditions in interteam frameworks as high trust perceptions should relax the explicitness of interteam formalities and boundaries - teams tend work off-contract to get tasks done (i.e., soft contracting). Informal compensating mechanisms like team-building, social networks and spontaneous work adjustment are typical soft contracting activities that characterize inter-team processes in high trust situations.

High distrust perceptions, however, should harden contract requirements because teams stipulate the details of their partnership. Many believe that stipulating future contingencies is impossible given the quick formation of teams working together to get work accomplished; however, many also believe that general contract work-around strategies can be identified a priori for resolving potential contract disputes. Swift forming teams in high distrust conditions should escalate the rigidity of inter-team interactions making them more formalized and bounded. 
Proposition 3: The higher trust perceptions are of future trading partners, the less formal and less bounded inter-team relationships tend to be.

Proposition 4: The higher distrust perceptions are of future trading partners, the more formal and more bounded inter-team relationships tend to be.

\section{Research Design}

\section{Methodology}

A qualitative, medium-term, scenario-based approach was employed where fifty student teams were exposed to four different scenarios in an outsourcing simulation (teams were divided nearly equally among the four scenarios). A medium-term simulation is one that takes more than one class day to do (i.e., a short-term approach) but less than a semester or quarter to complete (i.e. an extensive case study). This scenario approach took two weeks to collect data requiring four interview sessions with each team.

The time span in collecting this data took place over three years. The scenarios were designed to convey a realistic setting of trust and distrust. The simulation and results validating these scenarios are in Adler $(2005)^{1}$. Scenarios were presented to each consulting team that incorporated trust and distrust conceptualizations that were consistent with previous research in studying social effects in teams (Bond, Leung, \& Wan, 1982; Gomez, Shapiro, \& Kirkman, 2000).

To reduce the data and achieve some theoretical understanding, observers compared and discussed team perceptions with regard to how teams collectively viewed their colleague team. To assist reliability and validity of this data interpretation process, Miles and Huberman's (1984) procedures were followed as quotes were later transcribed and main themes were recorded in an ongoing journal. Following Pettigrew's (1990) lead, responses were coded for each team to ensure analytical themes were explicitly discussed, and data was reduced and presented accurately.

In addition, teams were informed that their responses may be used as part of this study and as such were urged to give an honest assessment of the simulation with regard to their own perceptions of team interactions (Glaser \& Strauss, 1967). Notes were hand-transcribed by observers at the end of each presentation to avoid interrupting the interaction of team members, and key words were put into tabular form to maintain the integrity of the data (Douglas, 1976; Miles \& Huberman, 1984). Team size typically ranged from four to six individuals. Observers used Table 1 based on Lewicki et al. (1998) and Rotter's (1980) frameworks to identify perceptions that fit one of the four scenarios.

We found consistent results in team perceptions within each scenario regarding how teams perceived a trust or distrust perception of their colleague team. Again, the purpose of this study was to measure how teams dealt with their colleague team based on perceptions of trust and distrust. Judgment calls had to be 
made early in the data reduction and interpretation of team perceptions, however, this is not uncommon for qualitative research (Glaser \& Strauss, 1967; Spradley, 1979). Observers threw out information that did not add to the purposes of our study. Issues like how information would be recorded and processed in teams, who would have ultimate decision authority, and team member roles, while important in other contexts, were not retained.

Individuals were also assessed after the simulation was completed with a written evaluation. The evaluation included questions ranging from how they would describe this simulation relative to how their teams parlayed perceptions into team interactions. Individuals were asked to compile a 'Lessons Learned' folder to advise future teams on how to deal with colleague teams in the four different scenarios. Individual answers were again analyzed for common factors to emerge as a result of the outsourcing simulation. At least two observers reviewed individual answers to document any inconsistencies between data collected through participant observation and interviewing.

\section{Simulation Background}

The complete outsourcing simulation used in this study can be found in Adler (2005). The researcher explained the scenario for Sam and Betty Construction (SBC) as follows: the draft work statement (e.g., a document that typically contains the contract requirements in a trading venture) and project definition worksheet (e.g., SBC's internal planning document) have been provided by the Swift Trust Corporation, a potential partner of $\mathrm{SBC}$, to form the basis for the partnership contract between the Swift Trust Corporation and SBC.

Observers divided individuals into teams and then assigned two teams to one of the four trust and distrust conditions listed in Table 1. Each of these two teams formed a pair of colleague teams. One team was responsible for revising the draft work statement and the other team was responsible for developing the project definition worksheet. Together, the teams must work to provide SBC a revised contract regarding how Swift Trust will provide architectural design support (see Table 2). Since Sam and Betty, co-owners of SBC, do not have much experience in architectural design, they have hired these two teams that specialize in project management. While the work statement is typically a binding document in the contract, the project definition worksheet is internally used at SBC to identify the major areas of work. Some work statement requirements are typically tied to work planned in the project definition worksheet but not all are included. For instance, SBC responsibilities might be more appropriate as a project definition worksheet item rather than a contractual document. The reason for this distinction might be that it would be more beneficial for SBC to have fewer formalized items in the contract. In other words, to reduce risk, SBC would make Swift Trust responsible for many things as in a high distrust situation. Thus, integration between the work statement and project definition work sheet is a must. Teams were observed and individuals were interviewed to better understand how a scenario would affect integration between teams. 
Table $1^{*}$

\section{Key Characteristics of Four Simultaneous Trust and Distrust Conditions}

\section{Characteristic}

\section{Low Trust/Low Distrust}
a. Casual acquaintances
b. Limited interdependence
c. Bounded, arms-length
d. Professional courtesy

\section{High Trust/Low Distrust}

a. High-value congruence

b. Interdependence promoted

c. Opportunities pursued

d. New initiatives

\section{Low Trust/High Distrust}

a. Undesirable eventualities expected and feared

b. Harmful motives assumed

c. Interdependence managed

d. Preemption; best offense is a good defense

e. Paranoia

\section{High Trust/High Distrust}

a. Trust but verify

b. Relationships highly segmented

c. Opportunities pursued and down-side risks/ vulnerabilities continually monitored 1a. Limited previous history so a new opportunity for everyone

Ib. Inputs and outputs between organization's processes are independent and not integrated

1c. Emotional relationship and transaction attachment between trading partners is not a factor to consider

1d. Respect for each other is expected and superficial

2a. Expectations between trading partners align

$2 b$. Sharing of work between organizations is expected

2 c. Creativity and innovation is an outcome of the partnership and valued by both partners

$2 d$. New strategies for maximizing the partnership are discussed openly and pursued by both parties

3a. History and reputational effects indicate the trading partner is not being honest in its relationship with your organization

$3 b$. Your organization is assumed to suffer in some way from this trading relationship

$3 \mathrm{c}$. Boundaries are set in all ways regarding access to your organization's information and resources

$3 d$. Penalties for abuse to contractual terms and conditions are strong enough to mitigate potential trading partner opportunism

3e. Worst case scenarios dominate your organization and team discussions regarding your potential trading partner

4a. Focus is a positive trading relationship but anticipate potential trading partner opportunism and limit as best as possible

4b. Partition information and resources are bounded to protect core competencies and discourage trading partner opportunism

$4 c$. Provide information sharing on team scouting and task coordination

“Adapted from Lewicki et al,'s (1998) framework 
Table 2

\section{Consulting Task Teams Role in the SBC and Swift Trust Partnership}

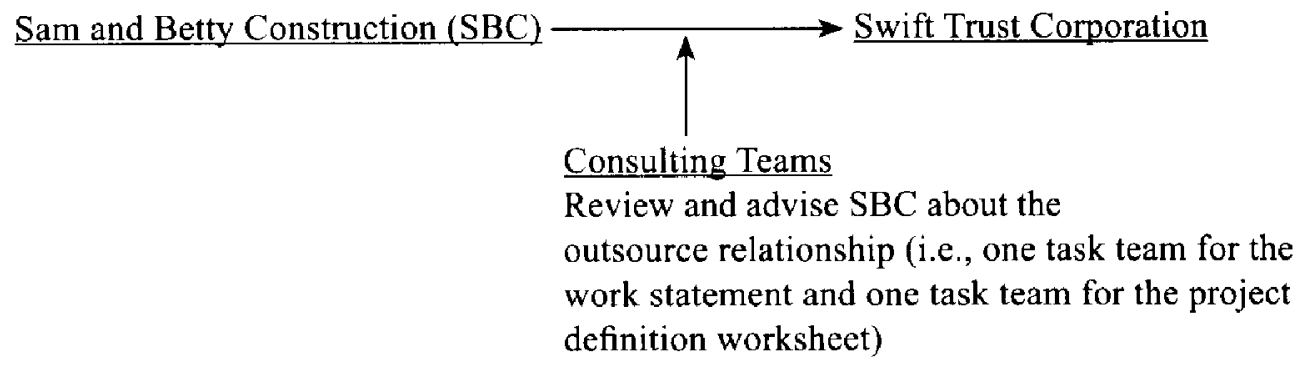

\section{Results}

In general, all propositions in this study were supported. Managing trust and distrust perceptions between teams while planning project work were viewed as difficult topics and tasks to do. Individuals stated that having colleague teams allowed them to better react to perceptions of trust and distrust more realistically. Summarized comments and observed outcomes in each trust and distrust condition are now discussed.

\section{Low Trust and Low Distrust}

The low trust and low distrust scenario represents a situation where teams struggle to define the partnership between SBC and the Swift Trust Corporation. The teams fall back on information provided in the simulation about whether a future trading partner might be worthy of trust or distrust. Many of the teams even go interview home builders outside of the simulation in hopes of better defining an actual case of low trust and distrust. Interactions between teams, thus, lead to little emotional buy-in since teams find little to commit to in the SBC-Swift Trust business relationship. As Lewicki et al. (1998) hypothesized, this is a situation where there is a lack of hope or confidence in the future trading partner.

As a consequence, teams assigned to this scenario typically gave simplistic presentations to their instructor since there is so little information on which to base a contract or planning document. Thus, teams varied greatly in the amount and quality of revisions to the work statement and project definition worksheet. Some teams invent requirements for the work statement assuming this is what was meant by the strategic leadership. An example is when a team adds work that is out of the original scope of the project. Many teams added work that had nothing to do with the original work statement in the simulation like adding a requirement to conduct a market survey of housing in other cities.

Outcomes for teams interacting in this scenario take on a 'checklist' mentality as teams struggle to 'fill in the blanks' by adding unnecessary project requirements into the contract. Not only does this make team interactions more potentially unnecessarily complex, it is also dangerous because these added interactions may 
create a degree of distrust between teams that is incommensurate with the novelty of their current trading relationship. Team outcomes that involve scope definition, communication planning, and leadership development revolve around keeping work independent and limited between SBC and Swift Trust. Team outcomes reflect a casualness to the SBC and Swift Trust trading relationship that leaves team members wanting more to the simulation and, ultimately, more from other teams with regard to team contributions.

\section{High Trust and Low Distrust}

The elements of high trust and low distrust are ones that have been proposed by researchers as the prototypical soft contracting scenario (Williamson \& Ouchi, 1981). High trust and low distrust typically lead to less emphasis on the contract and more emphasis on what teams really want in the SBC and Swift Trust partnership. Consequently, teams spend little time revising the work statement and project definition worksheet because of the generally held belief that Swift Trust could be relied upon. Even when teams identify known copyedit problems with the work statement, these grammar or typo errors were not corrected and allowed to remain in team outputs.

Team members stated that they really enjoyed working with their colleague team in this scenario as they learned a lot about outsourcing, strategy planning, and the difficulties of communicating requirements. This is not what teams generally said in other scenarios in this simulation. The positive experiences of individuals working in teams in this scenario support Proposition 1 because the heart of the matter is that when partners are viewed as trustworthy, team interactions reflect this positivism and work is viewed with a higher degree of quality.

Teams also typically explained that to address any particular issue all they would have to do is call Swift Trust and it could be worked out over the phone. Thus, inter-team interactions focused more on the content of the SBC and Swift Trust relationship emphasizing what really mattered not the legalities or formalities of the contracting framework. As one individual reflected about her team philosophy, "SBC has an open door policy with Swift Trust. Why wouldn't we trust them? We have a relationship with them that is effective in getting the job done." Thus, the high trust and low distrust condition leads to more informal governance between colleague teams suggesting soft contracting is where value is maximized and strategy is achieved. These results provided support for Proposition 3 where high trust situations lead to less bounded governance and formalization between teams.

\section{Low Trust and High Distrust}

The paranoiac view of a future business partner comes out clearly in this scenario supporting Lewicki et al.'s (1998) assertion that the low trust and high distrust condition leads to all kinds of negative fantasies. Thus, the focus of colleague teams is how to prevent Swift Trust from acting badly through the revision of work statement requirements to circumvent potential partner opportunism (i.e., 
transfer risk to Swift Trust when appropriate). Individuals discuss how difficult team discussions are in this scenario because most teams do not like thinking so negatively. Proposition 2 is supported because the high distrust in the SBC-Swift Trust partnership engenders a lower perceived quality to inter-team interactions. Team members take on an enforcement mentality that drains enthusiasm in interacting with their colleague team.

It should also come as no surprise that revisions to the work statement tend to be extensive and generally focused on putting absolute control into the hands of SBC management. Thus, Proposition 4 is supported as a trend towards hard contracting.

The absolute control aspect of this scenario is one key difference between it and the high trust and high distrust condition. For instance, in the draft work statement, adding "as accepted by SBC" to the end of the first sentence in paragraph 2.0 gives control to SBC. Also, in paragraph 6.0, the statement "the seller's project manager shall support SBC planning efforts by attending meetings, conferences, and reviews and responding to requests for information" is generally changed to "the seller's project manager shall support SBC planning efforts as SBC management dictates." These typical changes reflect the team's distrust of Swift Trust in honoring the terms and spirit of the partnership contract. Many teams also put penalty statements in the revised work statement. For instance, the penalty "failure to adequately identify all risk factors with accompanying assumptions makes this contract subject to termination by SBC" is a common type of sanction levied on Swift Trust if they do not comply.

As predicted by Lewicki et al. (1998), the work statement bounds the partnership taking on a very formal and arms-length transaction with Swift Trust. Inter-team interactions are also more rigid and difficult since teams have think of every negative situation, contemplate every possible contractual loophole, and never relax in their pessimism of Swift Trust. Colleague teams end up competing with each other to see who can identify the most loopholes and which loopholes might be the most egregious to SBC. As one individual stated, "We have to micromanage Swift Trust because they appear to not be able to manage themselves."

\section{High Trust and High Distrust}

The most common form of business relationship found today is the high trust and high distrust condition according to Lewicki et al. (1998). One reason for this is the complex and differentiated aspects of businesses where work is divided and delegated to teams to meet organizational objectives. Consequently, inter-team interactions result in detailed presentations and extensive revisions since not only does a team have to micro-manage Swift Trust's opportunism, they must also manage internal SBC work found in project definition worksheet. Teams in this scenario perceive degrees of low and high quality and formality and informality as predicted by Lewicki et al. (1998).

Individuals described the necessity of frequent communication and individual contribution, as Meyerson et al. (1996) noted, to the establishment of trust within 
the teams. Many individuals expressed frustration with their colleague team who did not actively participate in inter-team meetings and discussions. Propositions 1 through 4 were supported based on the degree to which team members made themselves available to discuss the complexities of the SBC and Swift Trust partnership. If team members made themselves available to meet, then they were viewed as being accountable and trust between teams grew. Inter-team interactions were generally viewed with higher quality. As one team member stated, "it is hard enough to figure out what is important between SBC and Swift Trust. Our colleague team, though, helped us figure out how to work around Swift Trust and present a good solution that met everyone's goals."

Relational contracting where teams operate through the social context to codify the SBC and Swift Trust partnership is clearly evident in this scenario. The binding together of each team's social contexts reflects a dynamic not evident in the other trust and distrust conditions. Team members learn how to manage suspicions and develop relationships simultaneously in managing business risk in outsourcing. For instance, if SBC loses money, financial risk is increased since SBC may not be able to pay Swift Trust for services rendered. If SBC loses competitive ground due to Swift Trust's lack of adequate advice, then Swift Trust may not retain future business relations with SBC. Teams struggled in how to protect both SBC and Swift Trust interests given the high trust and high distrust between the trading partners. This scenario adds some interesting strategic tension to the outsourcing decision where organizational and team boundaries become blurred and partnerships become more mature and complex.

\section{Discussion and Implications}

Using contracts, agreements, or other documents to formally bind organizations, divisions, and departments is a growing trend in organizations today. The outsourcing literature indicates that teams implement management decisions that have huge organizational implications (e.g., strategic ties; Ang \& Straub, 1998; Cheser, 1999). The use of team-based designs requires unique communication skills that many teams do not have nor do team members appreciate (Adler, 2004; Ancona \& Caldwell, 1992).

Teams often carry the responsibility for success or failure in implementing outsourcing decisions in provider selection and contract administration. Successful contract negotiation between outsource partners assumes teams can communicate effectively. The results of this study indicate that teams have different types of interactions when faced with different simultaneous trust and distrust perceptions. This study provides evidence that management of simultaneous trust and distrust issues are not limited to the formal organizational partnership but also to how teams implement outsourcing decisions.

The high trust and high distrust condition certainly represents many business relationships today. On an individual and team basis, many firms hire employees with a high trust and high distrust relationship either for reasons of security, 
or compartmentalization of access to parts of the firm, or for strategic human resource compliance in privacy or intellectual property protection. Employee identification cards serve as a reminder that firms highly trust employees to do their job, but that access to other work may not be part of their job. In this study, teams viewed giving colleague teams access only to the necessary parts of their assigned responsibilities on an 'as necessary' need to fulfill the partnership contract. What was interesting is that teams in this scenario needed confirmation from their colleague team since the simulation scenario was too complex to manage by themselves.

In addition, the high trust and low distrust scenario was indicative of teams where social context was the medium to get work done. Many businesses operate this way where a contract may be necessary to partner, but the contract is a mere formality to the partnership itself. Inter-team interactions represented low soft contracting characteristics in accordance with the Williamson and Ouchi (1981) framework.

The low trust and high distrust condition represented many situations where organizations are forced to partner due to necessity, not choice. This may be the result of a sole source environment, management compliance, or a monopoly amongst suppliers. In either case, the buyer partner is many times at the mercy of the supplier partner and thus, over time, abuses to this relationship become engrained into a distrust perception. Inter-team interactions took on a very competitive flavor as teams vied to be the winner in identifying the most loopholes or the loopholes with the largest potential damage to SBC.

Government contracting is a good example of this scenario. While not all government suppliers have done things to lead to distrust, many have, and once trust is violated, it is difficult to regain trust. The attempts by colleague teams to make SBC the absolute authority in the contract was surprising but lived up to the assumptions found in Lewicki et al.'s (1998) model when sometimes the best strategy is to make preemptive strikes against a unscrupulous partner. One can imagine a situation where the work statement would be negotiated by teams of lawyers regardless of the technical complexity of the project involved. Thus, in a low trust and high distrust condition, success may depend more on how good your lawyers are relative to a partner's lawyer(s). Loophole management becomes a critical skill in a high distrust and low trust scenario.

Many organizations have dealt with this issue by assigning multiple teams to review basic assumptions of every contract requirement. Other firms may take a more 'hands-off' approach relying on their future business partner to deliver whatever is required (e.g., soft contracting). Firms would be wise to challenge assumptions, expectations, and perceptions that lead to high and low trust and distrust when developing contract requirements.

\section{Implications to Researchers and Practitioners}

This research may be limited since business students were used as the source for this study. Commercial settings offer more legal maneuvering between orga- 
nizations and teams and, thus, more potential for altering perceptions collectively by team members. However, as Pettigrew (1990) notes, collecting and analyzing comparative data is a highly complex social and intellectual task. We believe that since this research was collected based on a validated outsourcing simulation used to investigate trust and distrust perceptions, our conclusions can be used in a variety of settings to understand how business relationships and team-based interactions occur.

Future research needs to consider top management's role in inter-team communication. A qualitative study of how trust and distrust perceptions change relative to organizational and team performance is also necessary and would be a logical extension of this research. This study is somewhat limited in that scenarios were used to simulate actual contract negotiations. Further research should also study teams in their real environments to either corroborate or refute these findings.

There are several implications for practitioners. This study highlights the need to understand external communication patterns of team designs. Teams must integrate a plethora of interests that include team perceptions about other teams and future business partners. Contrary to MacNeil's findings (1978), contracts, in some situations, are intentionally made more complete due to team distrust perceptions. Complete contracts may or may not be what organizations desire, or need, to fulfill strategic objectives like the outsourcing of work. Clearly, tactical implementation by teams is multifaceted requiring effective management oversight. A better understanding of external team communication pattern effects is fundamental for managing team personnel and assessing team performance.

\section{Conclusion}

A more robust understanding of the four scenarios in the Lewicki et al. (1998) model was gained in this research. Each of the scenarios can occur in business today and this study provides context in how teams interact based on differing combinations of trust and distrust. Feedback indicates that the simultaneous 'High Trust and High Distrust' situation characterizes a complex business environment that teams viewed as both a blessing and a curse. In other words, most respondents hoped for trust but settled for distrust in how they viewed colleagues, other teams, and potential business partners.

One interesting observation, in general, was the behavior of teams to mimic what they saw as the SBC and Swift Trust partnership. In other words, the framing of the SBC and Swift Trust business partnership greatly influenced the interteam interactions with the teams actually becoming part of the trust and distrust scenario they were asked to manage.

Perceptions of trust and distrust exist for many reasons and are affected by how teams interact within an organization. How teams deal with these and other contractual issues affects how well organizations fulfill organizational goals like outsourcing. Given the increasing demands placed upon managers today, a 
knowledge of how trust and distrust perceptions affect team interactions is fundamental to managing future business partnerships. Practical application of trust and distrust models must be further studied and developed as researchers have a duty to flesh out the factors that affect how teams frame information based on trust and distrust perceptions and how teams develop contractual requirements based on these perceptions. This medium-term, qualitative analysis provides insight into these demands.

\section{References}

Adler, T. (2004). A study of external team communication effects on team member perceptions of trust and distrust. Journal of Behavioral and Applied Management, $5(3), 245-279$.

Adler, T. (2005). The Swift Trust Partnership: A project management exercise investigating the effects trust and distrust in outsourcing relationships. Journal of Management Education, 29(5), 714-737.

Ancona, D., \& Caldwell, D. (1992). Bridging the boundary: External activity and performance in organizational teams. Administrative Science Quarterly, 37, 634-665.

Ang, S., \& Straub, D. (1998). Production and transaction economies and IS outsourcing: A study of the U.S. banking industry. MIS Quarterly, 22(4), 535-552.

Barthelemy, J., \& Adsit, D. (2003). The seven deadly sins of outsourcing. Academy of Management Executive, 17(2), 87-101.

Bazerman, C. (1983). Scientific writing as a social act: A review of the literature of thesociology of science. In P. V. Anderson, R. J. Brockman, \& C. R. Miller (Eds.), New essays in technical and scientific communication: Research, theory, practice. Farmingdale, NY: Baywood Publishing Co.

Bell, B. (1990). Two separate teams should be set up to facilitate strategic alliances. Journal of Business Strategies, 11, 63-65.

Benfratello, L., \& Sembenelli, A. (2002). Research joint ventures and firm level performance. Research Policy, 31(4), 493-507.

Bond, M., Leung, K., \& Wan, K. (1982). How does cultural collectivism operate? The impact of task and maintenance contributions on reward distribution. Journal of Cross-Cultural Psychology, 13, 186-200.

Boon, S., \& Holmes, J. (1991). The dynamics of interpersonal trust: Resolving uncertainty in the face of risk. In R. A. Hinde \& J. Grobel (Eds.). Cooperation and prosocial behavior (pp. 190-213), Cambridge: Cambridge University Press. 
Boswell, C. (2000). For Albany Molecular, proprietary technologies are key to success. Chemical Market Reporter, 258(21), 29-35.

Cheser, R. (1999). When teams go to war - against each other! Quality Progress, 32, 24-29.

Cohen, S., \& Bailey, D. (1997). What makes teams work: Group effectiveness research from the shop floor to the executive suite. Journal of Management, 23(3), 239-290.

Dasgupta, P. (1988). Trust as a commodity. In D. Gambetta (Ed.). Trust, Making and breaking cooperative relations. (pp. 44-72). Oxford: Blackwell.

Deutsch, M. (1960). The effect of motivational orientation upon trust and suspicion. $H u$ man Relations, 13, 123-139.

Douglas, J. (1976). Investigative social research: Individual and team field research. Beverly Hills, CA: Sage Publications, Inc.

Erickson, E. (1950). Childhood and Society. New York: Norton.

Glaser, B., \& Strauss, A. (1967). The discovery of grounded theory: Strategies for qualitative research. Chicago: Aldine.

Gomez, C., Shapiro, D., \& Kirkman, B. (2000). The impact of collectivism and in-group/ out-group membership on the evaluation generosity of team members. Academy of Management Journal, 43(6), 1097-1107.

Helewitz, J. (2003). The art of negotiation. Legal Assistant Today, 20, 66-68.

Hosmer, L. (1995). Trust: The connecting link between organizational theory and philosophical ethics. Academy of Management Review. 20, 379-403.

Jeffries, F., \& Reed, R. (2000). Trust and adaptation in relational contracting. Academy of Management Review, 25(4), 873-898.

Knorr, K., \& Knorr, D. (1978). From scenes to scripts: On the relationship between laboratory research and published paper in science. Research Memorandum No. 132. Vienna: Institute for Advanced Studies.

Lacity, M., \& Willcocks, L. (1998). An empirical investigation of information technology sourcing practices: Lessons from experience. MIS Quarterly, 22(3), 363-408.

Larson, C., \& LaFasto, F. (1989). Teamwork: What must go right/what can't go wrong. Newbury Park, CA: Sage.

Lewicki, R., McAllister, D., \& Bies, R. (1998). Trust and distrust: New relationships and realities. Academy of Management Review, 23(3), 438-458. 
Luhmann, N. (1979). Trust and power. Chichester, UK: Wiley.

MacNeil, I. (1978). Contracts: Adjustments of long-term economic relations under classical, neoclassical and relational contract law. Northwestern University Law Review, $72,854-906$.

Mayer, R., Davis, J., \& Schoorman, F. (1995). An integrative model of organizational trust. Academy of Management Review; 20(3), 709-734.

Meyerson, D., Weick, K., \& Kramer, R. (1996). Swift trust and temporary groups. In R. M. Kramer \& T. R. Tyler (Eds.), Trust in organizations: Frontiers of theory and research (pp. 166-195), Thousand Oaks, CA: Sage.

Miles, M., \& Huberman, A. (1984). Qualitative data analysis. Beverly Hills, CA: Sage Publications.

Mishra, A. (1996). Organizational responses to crisis: The centrality of trust. In R. M. Kramer, \& T. R. Tyler (Eds.), Trust in organizations: Frontiers of theory and research (pp. 261-287), Thousand Oaks, CA: Sage.

Niederman, F., Brangeay, J., \& Wetherbe, J. (1991). Information systems management issues for the 1990s. MIS Quarterly, 15(4), 474-500.

Paladino, M., Bates, H., \& da Silveira, G. (2002). Using a customer-focused approach to improve quality across the value chain: The case of Siderar. Total Quality Management, I3(5), 671-683.

Pettigrew, A. (1990). Longitudinal field research on change: Theory and practice, Organization Science, I(3), 267-292.

Robinson, W., \& Volkov, V. (1998). Supporting the negotiation life cycle. Communications of the ACM, 41, 95-103.

Rotter, J. (1980). A new scale for measurement of interpersonal trust. Journal of Personality. $35,651-665$.

Rousseau, D. (1995). Psychological contracts in organizations: Understanding written and unwritten agreements. Thousand Oaks, CA: Sage.

Schilling, M., \& Steensma, H. (2001). The use of modular organizational forms: An industry-level analysis. Academy of Management Journal, 44(6), 1149-1169.

Spradley, J. (1979). The ethnographic interview. NY: Holt, Rinehart \& Winston.

Williamson, O., \& Ouchi, W. (1981). The markets and hierarchies program of research: Origins, implications, prospects. In W. Joyce, \& A. Van de Ven (Eds.), Organizational Design. New York: Wiley. 
Zand, D. (1972). Trust and managerial problem solving. Administrative Science Quarterly. 17, 229-239.

Zuckerman, D., \& Higgins, M. (2002). Optimizing cross-organizational team performance and management. Pharmaceutical Technology, 26(6), 76-79.

\section{Footnote}

I Please contact the author for a full set of simulation materials

Terry Adler is currently Associate Professor of Management in the College of Business at New Mexico State University (NMSU). He received his Ph.D. in Strategy and Information Systems from the University of Cincinnati in 1996. Besides being widely published on such topics as trust and distrust and contracting issues, he was named the 2006 Donald C. Roush Professor of the Year. Dr. Adler has made over 200 international presentations to organizations throughout the world. 
